\title{
Earthworms and environment: a tool for diagnosis, assessment, monitoring, and remediation of soil pollution and soil quality
}

\author{
Yinsheng $\mathrm{Li}^{1} \cdot$ Branimir Hackenberger ${ }^{2}$ \\ Published online: 25 June 2020 \\ (C) Springer-Verlag GmbH Germany, part of Springer Nature 2020
}

This special issue of the Environmental Science and Pollution Research highlights selected papers presented at the 1st International Earthworm Congress (IEC1), which was held on June 24-29, 2018, in Shanghai, China.

This congress incorporated the 11th International Symposium on Earthworm Ecology (ISEE 11), the 8th International Oligochaeta Taxonomy Meeting (IOTM 8), and the 1st International Earthworm Industry Forum (IEIF 1), which is the first time to integrate these meetings. The presentations were organised in six sessions on the following topics: (1) Earthworm taxonomy and systematics; (2) Earthworm phylogeny and evolution; (3) Global environmental change and earthworms; (4) Earthworm ecotoxicology; (5) Earthworms and agriculture-soil, waste, and animal breeding; (6) Earthworms and medicine. The intention of organising and scientific committees was to bring together the talents and present the frontiers of knowledge in all fields of science and technology involving earthworms.

Earthworms are well known to play an important role in different processes in terrestrial ecosystems and present a major component of soil fauna communities. To better understand earthworms' impact on the environment, it is important

Responsible Editor: Philippe Garrigues

Yinsheng Li

yinshengli@sjtu.edu.cn

Branimir Hackenberger

hackenberger@biologija.unios.hr

1 School of Agriculture and Biology, Shanghai Jiao Tong University, 800, Dongchuan Road, Shanghai 200240, China

2 Subdepartment of Quantitative Ecology, Department of Biology, Josip Juraj Strossmayer University, Osijek Cara Hadrijana 8A, HR-31000 Osijek, Croatia to study their life cycles and physiology of different species under various environmental conditions. Furthermore, earthworms are rather sensitive and are used as test organisms to assess the impact of xenobiotics and changed environmental conditions from molecular to population levels. Due to the benefits they can provide, earthworms are also utilised in ecosystem management - in soil remediation, waste management, land reclamation, etc. Additionally, the earthworms are also used as animal feed and medicine. Besides fruitful discussions and interesting presentations, the major stronghold of IEC1 is the cognition that human beings will continue to benefit from the development of earthworms' resources and preservation of their biodiversity and, consequently, soil health and resilience.

This special issue of the Environmental Science and Pollution Research presents 13 independent peerreviewed papers providing some examples of the IEC1 achievements and results. This special issue cannot fully reflect the diversity and creativity of the ideas and new insights that were shared at IEC1. However, as editors, we hope that this issue may prompt scientists from the diverse fields to participate in earthworm research to come: the collected papers show and justify the strong position of the knowledge about earthworms as a tool for the diagnosis, assessment, monitoring, and remediation of soil pollution and soil quality in worldwide studies.

\footnotetext{
Acknowledgements The guest editors wish to thank Philippe Garrigues, editor-in-chief of the Environmental Science and Pollution Research, as well as Florence Delavaud and Fanny Creusot for their advice and support during the preparation of this special issue. We also thank the anonymous reviewers for their useful and timely comments on the papers submitted to this issue.
}

Publisher's note Springer Nature remains neutral with regard to jurisdictional claims in published maps and institutional affiliations. 


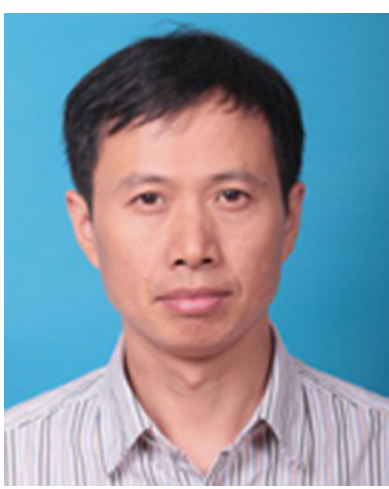

Yinsheng $\mathbf{L i}$ is a full professor of Department of Resource \& Environment, School of Agriculture and Biology, Shanghai Jiao Tong University. His main research topics are related to soil and earthworm, specifically, (1) soil ecotoxicology: ecological toxicology and pollution control of drug residues, drugresistant gene, and heavy metals; (2) earthworm biotechnology: research and development of earthworm antibacterial peptides; treatment of organic waste (livestock

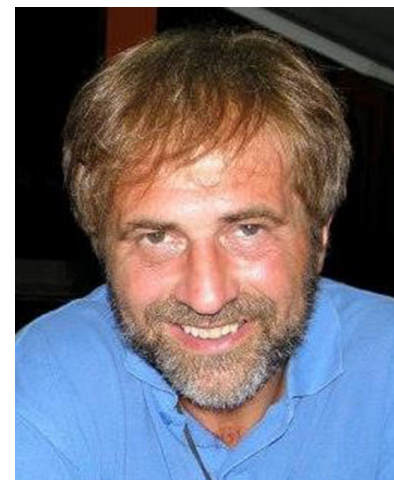

Branimir Hackenberger is a full professor of Department of Biology at J. J. Strossmayer University in Osijek, Croatia. His main research topics are related to (1) soil ecotoxicology, the effects of different xenobiotics on earthworms, enchytraeids, and collembola; and (2) biostatistics, environmental bioinformatics, and computational toxicology. He has (co)authored more than 80 papers in international journals and conference proceedings.

manure, sludge, straw, kitchen waste) by earthworms and microbes; soil amelioration and bioremediation. He (co)authors about 60 papers in international journals and conference proceedings on these topics. 